\title{
Effect of shell structure on the fission of sub-lead nuclei
}

\author{
Guillaume Scamps ๑* \\ Center for Computational Sciences, University of Tsukuba, Tsukuba 305-8571, Japan \\ and Institut d'Astronomie et d'Astrophysique, UniversitLibre de Bruxelles, Campus de la Plaine CP 226, BE-1050 Brussels, Belgium \\ Cédric Simenel ${ }^{\dagger}$ \\ Department of Theoretical Physics and Department of Nuclear Physics, Research School of Physics and Engineering, \\ Australian National University, Canberra, Australian Capital Territory 2601, Australia
}

(Received 2 April 2019; revised manuscript received 11 June 2019; published 28 October 2019)

\begin{abstract}
Fission of atomic nuclei often produces mass asymmetric fragments. However, the origin of this asymmetry was believed to be different in actinides and in the sub-lead region [Andreyev et al., Phys. Rev. Lett. 105, 252502 (2010)]. It has recently been argued that quantum shell effects stabilizing pear shapes of the fission fragments could explain the observed asymmetries in fission of actinides [Scamps and Simenel, Nature 564, 382 (2018)]. This interpretation is tested in the sub-lead region using microscopic mean-field calculations of fission based on the Hartree-Fock approach with BCS pairing correlations. The evolution of the number of protons and neutrons in asymmetric fragments of mercury isotope fissions is interpreted in terms of deformed shell gaps in the fragments. A new method is proposed to investigate the dominant shell effects in the prefragments at scission. We conclude that the mechanisms responsible for asymmetric fissions in the sub-lead region are the same as in the actinide region, which is a strong indication of their universality.
\end{abstract}

DOI: 10.1103/PhysRevC.100.041602

Nuclear fission was discovered in 1938 by bombarding ${ }^{235} \mathrm{U}$ with slow neutrons, producing a heavy fragment in the barium region and a light one near krypton $[1,2]$. Most of the questions opened by this fundamental discovery are still being debated 80 years later $[3,4]$. In particular, the origin of the observed mass asymmetry between the fragments is interesting as it offers a unique signature of quantum effects in large-amplitude collective motion.

The spherical shell model developed by Mayer in 1950 [5] explains the extra stability of nuclei with so-called magic numbers of protons and neutrons associated with fully occupied quantum shells (analogous to noble gas in atomic physics). Closed shells have then been naturally invoked as possible drivers to asymmetric fission [6-10], energetically favoring the formation of fragments with (doubly) magic clusters such as ${ }_{50}^{132} \mathrm{Sn}_{82}$. Neutron deformed shell effects in fission fragments with $N \approx 88$ neutrons [11] as well as in the fissioning nucleus [12] have also been invoked. However, experiments show that the main driver to asymmetric fission in the actinide region is the number of protons of the heavy fragment, which remains particularly stable around $Z \approx 54$ [13-15].

We recently proposed a possible explanation for this stability [16] based on octupole (pear shape) deformed shell effects

\footnotetext{
*gscamps@ulb.ac.be

†cedric.simenel@anu.edu.au
}

in the ${ }^{144} \mathrm{Ba}$ region $[17,18]$. The latter are induced by energy gaps at $Z=52$ and 56 for a combination of quadrupole (cigar shape) and octupole deformations [19]. Just before scission, the prefragments are connected by a neck which enforces their octupole deformation due to a combination of short-range nuclear attraction and long-range Coulomb repulsion. The production of nuclei like ${ }^{144} \mathrm{Ba}$ which can exhibit octupole shapes for no or little cost in energy is then naturally favored (unlike ${ }^{132} \mathrm{Sn}$ which is hard to deform). This mechanism offers an explanation for mass asymmetric fission in actinides. However, the question of its universality remains open, i.e., can it explain asymmetric fission in other regions of the nuclear chart?

A new region of asymmetric fission has been discovered more recently in the sub-lead region [20] and actively studied experimentally by several groups [21-26]. In particular, ${ }^{180} \mathrm{Hg}$ was found to fission asymmetrically, with heavy and light fragment mass distributions centered around $A \approx 100$ and 80 nucleons, respectively, while its fission was expected to be symmetric due to closed spherical shells in ${ }_{40}^{90} \mathrm{Zr}_{50}$ [20]. It was then referred to as a "new type of asymmetric fission," because the observed asymmetry could clearly not be explained by spherical shell effects. Theoretically, this could reflect the presence of an asymmetric saddle point with a ridge between symmetric and asymmetric fission valleys [20,2729]. Different explanations were proposed, involving shell effects in prescission configurations associated with dinuclear structures [30], or with quadrupole deformed neutron shells in the fragments [31-35] as well as in the fissioning nucleus [36]. 
In this Rapid Communication, we show that the mechanism based on octupole deformed shell effects in the fragments, which we invoked to interpret asymmetric fission in actinides [16], also plays an important role in the sub-lead region. We first focus our theoretical analysis on ${ }^{180} \mathrm{Hg}$. We then investigate the evolution of asymmetric fission across the mercury isotopic chain. We finally present a comparison of predicted asymmetric modes with existing experimental data in the sub-lead region.

Our theoretical analysis is based on the Hartree-Fock (HF) self-consistent mean-field theory (or energy density functional approach) with BCS pairing correlations. This microscopic approach has been successfully used by several groups with various levels of sophistication to investigate both static [37-45] and dynamical [16,46-53] characteristics of fissioning nuclei (see also Ref. [54] for a recent review).

Here we choose a static approach. Indeed, for the sub-lead nuclei studied here, we do not expect dynamical effects to play a major role as their fission valleys do not exhibit long descent of the potential from saddle point to scission (see Fig. 1 and Supplemental Material [55], Fig. 2). This is at variance with the actinide region in which dynamical effects occurring during the descent of the potential from saddle to scission may impact the outcome of the fission process (e.g., the total kinetic energy of the fragments [46]). Nevertheless, predictions of time-dependent and static approaches are in relatively good agreement in terms of the fragment mass and charge asymmetry. Furthermore, for the systems we have studied in the sub-lead region, we found that the saddle and scission points are much closer than in actinides. Thus, the dynamical effects are not expected to induce strong deviations from results obtained in a quasistatic picture. We therefore conclude that the mechanisms responsible for mass-asymmetric fission in the sub-lead region can be studied with a time-independent microscopic approach.

The constrained Hartree-Fock method with the BCS approximation for the pairing correlations is used. The nuclear interaction is described by the SLy4d parametrization of the Skyrme energy density functional (EDF) [56], with a surface-type pairing functional with interaction strength $V_{0}^{n n}=1256 \mathrm{MeV} \mathrm{fm}^{3}$ and $V^{p p}=1462 \mathrm{MeV} \mathrm{fm}^{3}$ [57]. The calculations are done with a modified version of the EV8 solver [58] where only one plane of symmetry is used. A spatial grid of dimension $L_{x} \times L_{y} \times 2 L_{z}=40 \times 19.2 \times 19.2 \mathrm{fm}^{3}$ with a mesh spacing of $0.8 \mathrm{fm}$ is used.

To study a large number of systems with a unique method, the following procedure has been applied. First, a constrained calculation is done with a quadrupole constraint $Q_{20}=47.3 \mathrm{~b}$ and several octupole constraints from $Q_{30}=0$ to $22 \mathrm{~b}^{3 / 2}$. The octupole constraint is then released letting the system explore the bottom of the fission valley(s). Then the valley potential energy curves are determined by making small evolutions of the quadrupole constraint from $Q_{20}=47.3$ to $0 \mathrm{~b}$ and from $Q_{20}=47.3$ to $110 \mathrm{~b}$.

It is also important to note that our fission valleys are obtained at zero temperature. Modification of the potential energy surface at finite temperature can indeed have an effect

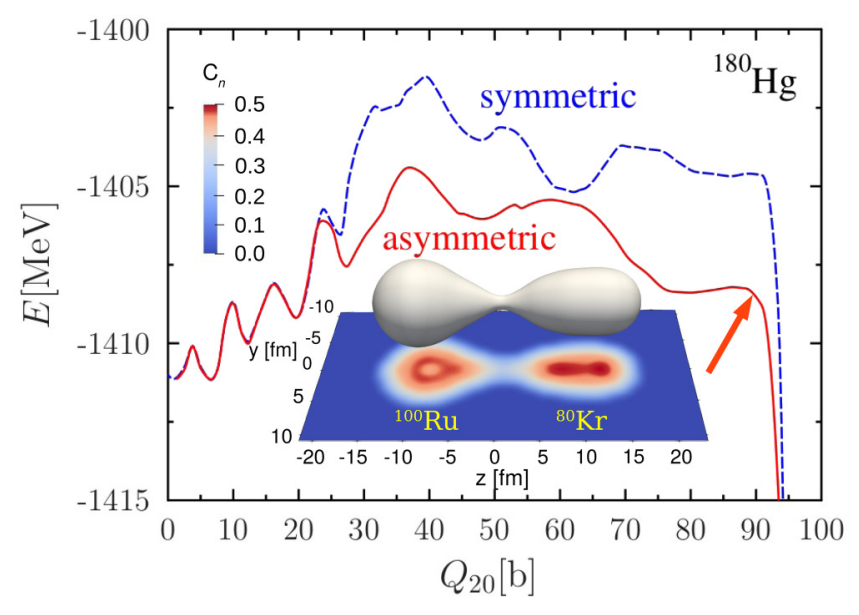

FIG. 1. Potential energy as a function of quadrupole moment along the symmetric fission path (dashed line) and asymmetric fission valley (solid line) of ${ }^{180} \mathrm{Hg}$. Isodensity surface at half saturation density $\rho_{0} / 2=0.08 \mathrm{fm}^{-3}$ and neutron localization function (projection) are shown for ${ }^{180} \mathrm{Hg}$ with quadrupole moment $Q_{20}=90$ $\mathrm{b}$, just before scission in the asymmetric fission valley, as indicated by the red arrow.

on the asymmetry of the fragment mass distribution [59-61]. However, self-consistent calculations indicate that fission modes are expected to be weakly influenced by excitation energy in the mercury region [44].

This approach allows us to study the shape of a system undergoing fission, and in particular to investigate the role of shell effects in the fragments. We also use it to predict the average number of protons and neutrons in the fragments for a given fission mode. A direct comparison can then be made with the centroids of experimental fission fragment mass distributions for asymmetric modes. Widths and shapes of these distributions, as well as a quantitative study of the competition between symmetric and asymmetric modes, are beyond the scope of this work and would require a more advanced treatment of fluctuations via, e.g., the time-dependent generator coordinate method [59-64], stochastic dynamics on top of a potential energy surface $[28,65,66]$, or stochastic mean-field calculations [67].

Figure 1 shows the evolution of the potential energy as a function of quadrupole moment for a symmetric path and in the asymmetric valley. The fact that the asymmetric valley remains significantly lower in energy than the symmetric path is a clear indication that asymmetric fission is energetically favored in this system. The predicted outcome of the ${ }^{180} \mathrm{Hg}$ asymmetric fission is a light fragment centered around ${ }_{36}^{80} \mathrm{Kr}_{44}$ and a heavy one around ${ }_{44}^{100} \mathrm{Ru}_{56}$, in excellent agreement with the masses observed experimentally [20].

The isodensity surface plotted in Fig. 1 shows that both fragments have significant quadrupole and octupole deformations. The neutron localization function $[68,69]$ (see Supplemental Material [55]) which is shown as a projection in Fig. 1 also exhibits strong quadrupole and octupole shapes within the prefragments. At scission, the associated quadrupole 

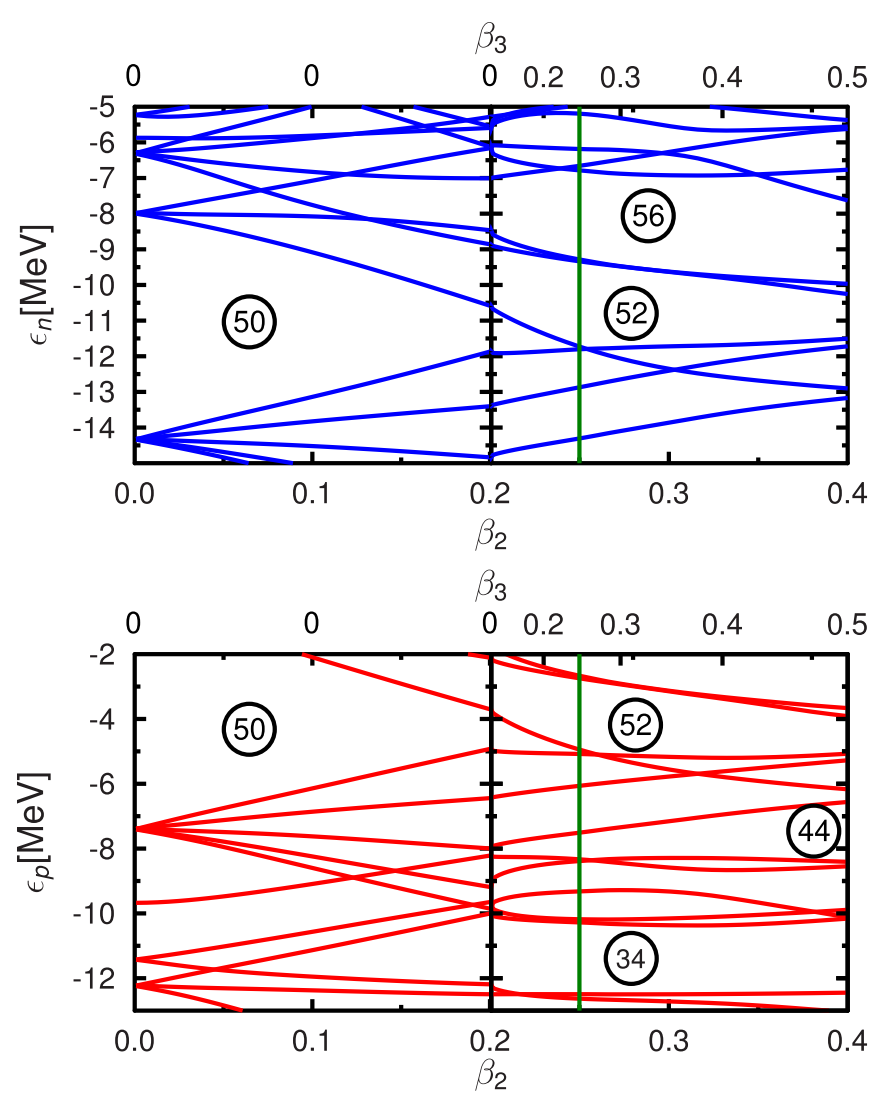

FIG. 2. Neutron (top) and proton (bottom) single-particle energies as a function of the quadrupole (lower scale) and octupole (upper scale) deformation parameters in ${ }^{100} \mathrm{Ru}$ (heavy fragment in ${ }^{180} \mathrm{Hg}$ fission). The left panel is obtained with an octupole constraint $\beta_{3}=0$. The right panel is obtained by constraining $\beta_{3}$ and without constraint on $\beta_{2}$.

deformation parameters are $\beta_{2}^{L} \simeq 0.75$ and $\beta_{2}^{H} \simeq 0.25$ for ${ }^{80} \mathrm{Kr}$ and ${ }^{100} \mathrm{Ru}$ fragments, respectively, indicating a compact heavy fragment and an elongated light one. The octupole deformation parameters are $\beta_{3}^{L} \simeq \beta_{3}^{H} \simeq 0.25$ for both fragments. These octupole deformations are not surprising, as pear shapes are induced by the neck in which strong nuclear attraction between the prefragments is still present. Therefore, the formation of fragments with small octupole deformation energy (and even stable octupole shapes) is expected to be energetically favored. Conversely, the formation of nuclei which are hard to deform should be hindered.

$\mathrm{HF}+\mathrm{BCS}$ calculations have been performed with quadrupole and octupole deformation constraints to investigate deformed shell effects in the fragments. Figure 2 shows the evolution of single-particle energies $\epsilon_{n}$ and $\epsilon_{p}$ in ${ }^{100} \mathrm{Ru}$. The left panel is obtained by varying $\beta_{2}$ and with $\beta_{3}=0$. The right panel is obtained by varying $\beta_{3}$ without quadrupole constraint. In this case, energy minimization with increasing $\beta_{3}$ leads to increasing $\beta_{2}$ as well (Fig. 2, right). Spherical gaps (magic numbers) at $\beta_{2,3}=0$ disappear with quadrupole deformation, while new deformed gaps appear. In particular, large gaps at proton and neutron numbers 52 and 56 (compact fragment) are observed at the quadrupole and octupole deformations of the fragments at scission (green solid lines).

These shell gaps are present for a broad range of octupole deformations thanks to octupole correlations. The origin of octupole correlations in nuclei has been widely discussed in the literature [70-72]. In fact, these octupole correlations for $Z=52$ and 56 protons [19] are expected to favor the formation of the heavy fragment (and thus drive asymmetry) in fission of actinides [16]. Comparing these numbers with those of neutrons in the fission fragments of ${ }^{180} \mathrm{Hg}$, we see that the large gap $N=56$ (Fig. 2) is indeed expected to favor the formation of ${ }_{44}^{100} \mathrm{Ru}_{56}$, and can therefore explain the asymmetry observed in ${ }^{180} \mathrm{Hg}$ fission.

Deformed shell effects could also be present in the light fragment. We see in Fig. 1 that the ${ }^{80} \mathrm{Kr}$ prefragment is strongly elongated $\left(\beta_{2}^{L} \simeq 0.75\right)$, in addition to its octupole deformation. Shell gaps are often observed in single-particle spectra at large $\beta_{2}$, e.g., for $32-36$ or $42-46$ protons or neutrons (see, e.g., [73] and Supplemental Material [55]). However, to affect fission, these gaps must also be stable against a range of octupole deformations. The method used above to identify shell gaps using an isolated deformed fragment can be difficult to apply for large deformations since a combination of octupole and large quadrupole constraints reproduce the shape of the prefragment only approximatively.

We now introduce a complementary method to investigate shell effects in the prefragments. Instead of constraining the shape of an isolated fragment, the single-particle energies are studied in the entire fissioning nucleus to search for the appearance of energy gaps near scission. The main difficulty here is to assign the level to one fragment or to the other.
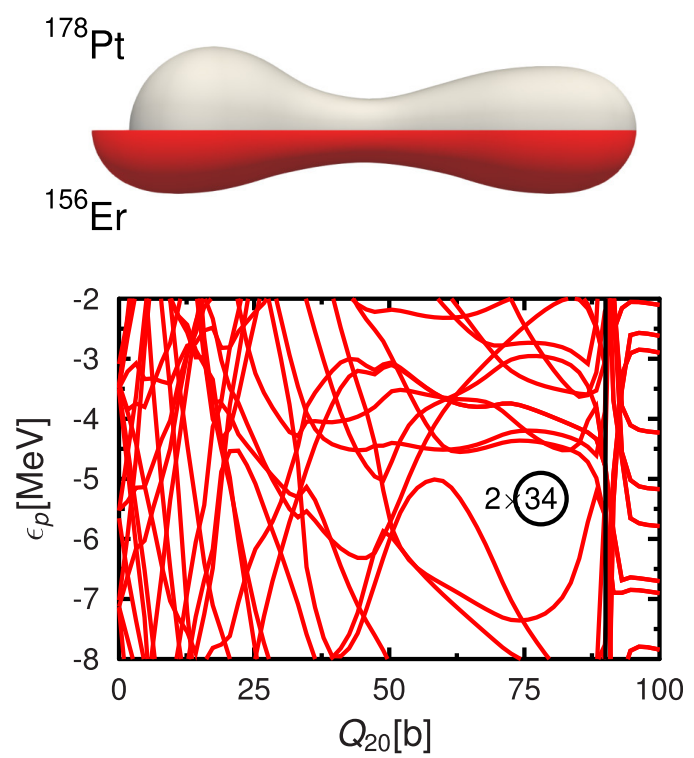

FIG. 3. Top: Comparison between the isodensity surface of the ${ }^{178} \mathrm{Pt}$ at deformation $Q 2=80 \mathrm{~b}$ and the ${ }^{156} \mathrm{Er}$ at deformation $Q 2=$ 82 b. Bottom: Proton single-particle energies as a function of the quadrupole (lower left scale) deformation parameters in the ${ }^{156} \mathrm{Er}$ fissioning system $(Z=2 \times 34)$. The black vertical line marks the position of the scission configuration. 


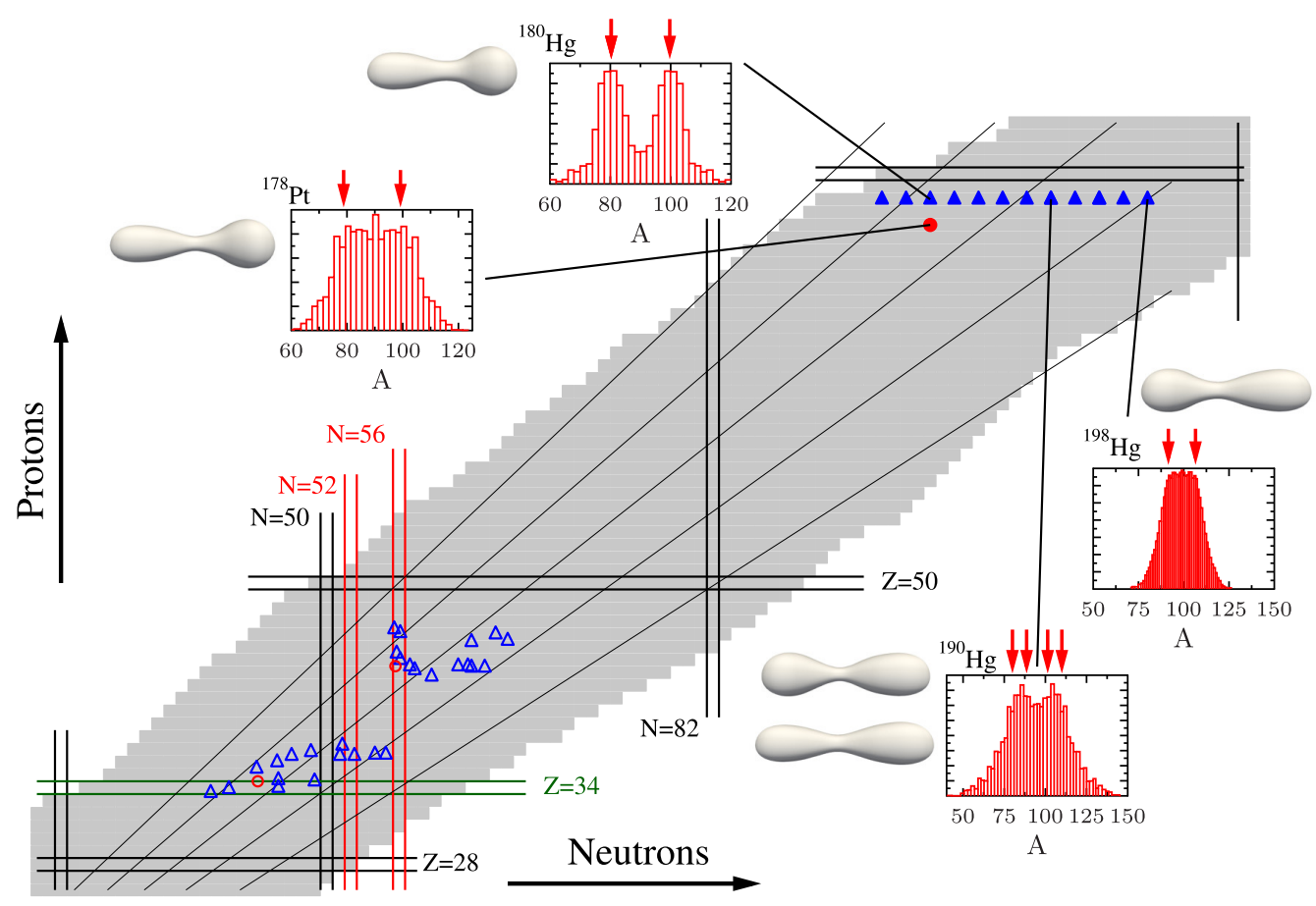

FIG. 4. Nuclear chart with spherical magic numbers (black solid lines) and main deformed shell gaps affecting fission of sub-lead nuclei (colored solid lines). Asymmetric fission properties of mercury isotopes (full triangles) and ${ }^{178} \mathrm{Pt}$ (full circle) have been studied with constrained $\mathrm{HF}+\mathrm{BCS}$ calculations. Expected centroids of light and heavy fragments are shown with associated open symbols. Insets show a comparison of theoretical predictions (arrows) with experimental data (histograms) from Refs. [20,22,74,75]. Isodensities surface at half the saturation density $\rho_{0} / 2=0.08 \mathrm{fm}^{-3}$ at scission associated with each inset are shown for each asymmetric fission mode with the heavy fragment on the right.

However, this is not a problem if the fission is symmetric since in this case the shell gap is present in both fragments; e.g., if there is a gap for $Z$ protons in the prefragment, we expect to see a gap for $2 Z$ protons in the total system [46]. Assuming that the shape of the prefragment does not depend strongly on the fissioning system, we can substitute an asymmetric fission $A \rightarrow B+C$ to a symmetric one $D \rightarrow B+B$. This method has the advantage of disentangling the shell structure of the two fragments $\mathrm{B}$ and $\mathrm{C}$.

Figure 3 illustrates the method for the proton levels in the symmetric fission ${ }_{68}^{156} \mathrm{Er} \rightarrow{ }_{34}^{78} \mathrm{Se}+{ }_{34}^{78} \mathrm{Se}$. The presence of a shell gap at $2 \times 34$ indicates a possible enhancement of the formation of this fragment, including in non-asymmetric fissions. Although the light fragment in ${ }^{180} \mathrm{Hg}$ asymmetric fission has $Z=36$, it is possible that it gains some stability thanks to the $Z=34$ deformed gap. Note that the shape of the light prefragment in ${ }^{180} \mathrm{Hg}$ or ${ }^{178} \mathrm{Pt}$ asymmetric fission and in ${ }^{156} \mathrm{Er}$ symmetric fission are very similar, as shown by the isodensity surfaces in the top of Fig. 3. Thus, they are expected to have a similar shell structure.

Asymmetric fissions of mercury isotopes with an even number of nucleons ranging from $A=176$ to $A=198$ have been studied with these techniques. The predicted average fragments are shown on the nuclear chart in Fig. 4 (open triangles) and given in [55]. Each of the most neutron deficient isotopes ${ }^{176-184} \mathrm{Hg}$ produces a compact heavy fragment with $N_{H} \simeq 56$, while each of the less neutron deficient isotopes
${ }^{190-198} \mathrm{Hg}$ has a light compact fragment with $N_{L} \simeq 51-55$ in which octupole shapes are favored by the deformed gaps at $N=52$ and 56. Note that these deformed shell effects are also expected to affect symmetric fission in ${ }^{184-192} \mathrm{Hg}$ with production of fragments having 52 to 56 neutrons (see, e.g., isodensity of ${ }^{188} \mathrm{Hg}$ symmetric fission near scission in Supplemental Material [55], Fig. 1). In addition to mercury isotopes, asymmetric fission of $A \simeq 178$ isobars has also been studied and found to form compact heavy fragments with $N \simeq$ 54-58 neutrons, thus confirming the persistence of $N=56$ octupole deformed shell effects away from mercury isotopes (see [55]).

Elongated shell gaps are also observed that could influence the fission of mercury isotopes. The shell gap $Z=34$ (see Fig. 3) is expected to play a role in the formation of the light fragments in ${ }^{176-194} \mathrm{Hg}$ asymmetric fission. Interestingly, we found a second asymmetric valley in ${ }^{190,192,194} \mathrm{Hg}$ with $Z_{L} \simeq$ 34 elongated fragments. All heavy fragments in ${ }^{188-198} \mathrm{Hg}$ asymmetric fissions are elongated with $Z_{H} \simeq 42-46$ protons. This indicates a possible influence of quadrupole shell gap at $\beta_{2}>0.5$ with $42-46$ protons [73,76] (see Supplemental Material [55], Fig. 3).

In Fig. 5, we show the energy that is required to deform a nucleus from the spherical shape to the typical deformation of the fragments at scission in the case of the compact $\left(\beta_{2,3}=0.25\right)$ and elongated $\left(\beta_{2}=0.8\right.$ and $\left.\beta_{3}=0.25\right)$ shapes. The positions of the fragments on the nuclear chart 

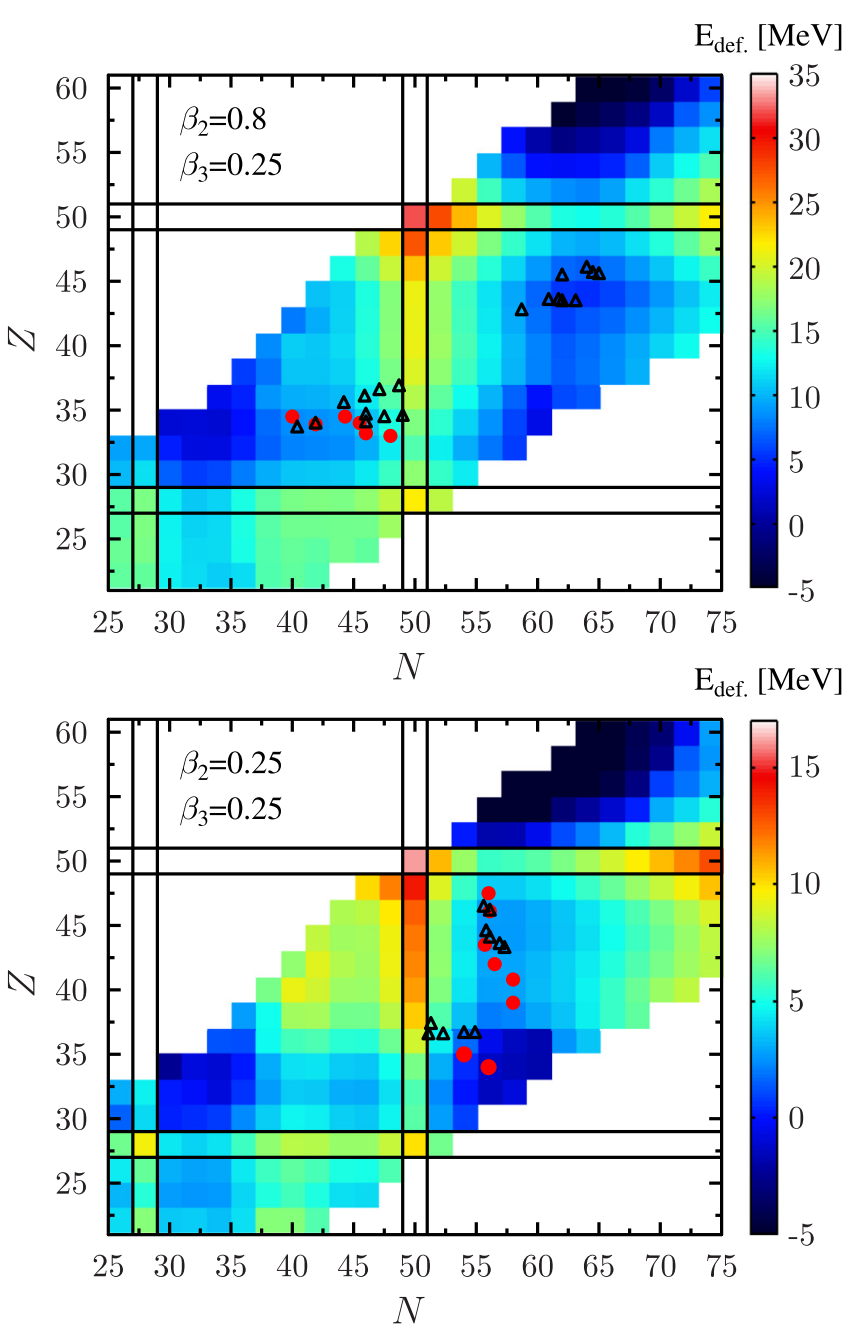

FIG. 5. Deformation energy in even-even nuclei (background color) for a constrained deformation corresponding to the elongated (top) and to the compact (bottom) shapes with $\left\{\beta_{2}=0.8, \beta_{3}=0.25\right\}$ and $\left\{\beta_{2}=0.25, \beta_{3}=0.25\right\}$, respectively. The expected values of the neutron and proton numbers of the fission fragments in the asymmetric mode of systems from Supplemental Material [55], Table I (black triangles) and Supplemental Material [55], Table II (red dots) are shown if the fission fragments have approximatively the deformations used to generate the background figure. are found in the area for which the deformation energy is small.

Finally, our theoretical predictions are compared with available experimental mass distributions in ${ }^{180,190,198} \mathrm{Hg}$ and ${ }^{178} \mathrm{Pt}$, shown in insets in Fig. 4. All these nuclei exhibit various level of asymmetry in their experimental fission fragment mass distributions. Although more data are required to achieve definitive conclusions, it is encouraging to see that the predicted asymmetric modes are all compatible with the experimental distributions.

Asymmetric fission has been studied theoretically in the sub-lead region. Its origin is interpreted as an effect of octupole correlations induced by deformed shell gaps at $N=$ 52-56 neutrons in the fission fragments (or prefragments), although shell gaps associated with large quadrupole deformations at 34 and $42-46$ protons seem to also contribute. Similar effects being present in actinide fission, we therefore conclude that the mechanisms driving mass-asymmetric fission are the same in both regions. It would be interesting to investigate the impact of octupole correlations in fission of superheavy elements (SHEs), in which shell effects in ${ }^{208} \mathrm{~Pb}$ could induce superasymmetric fission [77-81]. Similar effects have also been predicted [82-86] and observed [82,87] in quasifission. Despite being doubly magic, ${ }^{208} \mathrm{~Pb}$ has a low-lying collective octupole vibrational state and could thus be formed as a fission fragment with a pear shape (as shown in [55]).

Discussions with D. J. Hinde, C. Schmitt, B. Jurado, A. Chatillon, and W. Nazarewicz are acknowledged. This work has been supported by the Australian Research Council under Grants No. DP160101254 and No. DP190100256. The calculations have been performed in part at the NCI National Facility in Canberra, Australia, which is supported by the Australian Commonwealth Government, in part using the COMA system at the CCS in University of Tsukuba supported by the HPCI Systems Research Projects (Project ID hp180041), and using the Oakforest-PACS at the JCAHPC in Tokyo supported in part by Multidisciplinary Cooperative Research Program in CCS, University of Tsukuba. This work was supported by the Fonds de la Recherche Scientifique (F.R.S.-FNRS) and the Fonds Wetenschappelijk Onderzoek - Vlaanderen (FWO) under the EOS Project nr O022818F.
[1] L. Meitner and O. R. Frisch, Nature (London) 143, 239 (1939).

[2] O. Hahn and F. Strassmann, Naturwissenschaften 27, 11 (1939).

[3] K.-H. Schmidt and B. Jurado, Rep. Prog. Phys. 81, 106301 (2018).

[4] A. N. Andreyev, K. Nishio, and K.-H. Schmidt, Rep. Prog. Phys. 81, 016301 (2018)

[5] M. Mayer, Phys. Rev. 78, 22 (1950).

[6] M. G. Mayer, Phys. Rev. 74, 235 (1948).

[7] L. Meitner, Nature 165, 561 (1950).

[8] H. Faissner and K. Wildermuth, Nucl. Phys. 58, 177 (1964).

[9] C. L. Zhang, B. Schuetrumpf, and W. Nazarewicz, Phys. Rev. C 94, 064323 (2016).
[10] J. Sadhukhan, C. Zhang, W. Nazarewicz, and N. Schunck, Phys. Rev. C 96, 061301(R) (2017).

[11] B. D. Wilkins, E. P. Steinberg, and R. R. Chasman, Phys. Rev. C 14, 1832 (1976).

[12] C. Gustafsson, P. Möller, and S. Nilsson, Phys. Lett. B 34, 349 (1971).

[13] J. P. Unik, L. E. Glendenin, K. F. Flynn, A. Gorski, and R. K. Sjoblom, in IAEA Third Symposium on Physics and Chemistry of Fission, Vol. II (IAEA, Vienna, 1974) pp. 19-45.

[14] K.-H. Schmidt, S. Steinhäuser, C. Böckstiegel, A. Grewe, A. Heinz, A. R. Junghans, J. Benlliure, H.-G. Clerc, M. de Jong, J. Müller, M. Pfützner, and B. Voss, Nucl. Phys. A 665, 221 (2000) 
[15] C. Böckstiegel, S. Steinhäuser, K.-H. Schmidt, H.-G. Clerc, A. Grewe, A. Heinz, M. de Jong, A. R. Junghans, J. Müller, and B. Voss, Nucl. Phys. A 802, 12 (2008).

[16] G. Scamps and C. Simenel, Nature 564, 382 (2018).

[17] B. Bucher, S. Zhu, C. Y. Wu, R. V. F. Janssens, D. Cline, A. B. Hayes, M. Albers, A. D. Ayangeakaa, P. A. Butler, C. M. Campbell, M. P. Carpenter, C. J. Chiara, J. A. Clark, H. L. Crawford, M. Cromaz, H. M. David, C. Dickerson, E. T. Gregor, J. Harker, C. R. Hoffman, B. P. Kay, F. G. Kondev, A. Korichi, T. Lauritsen, A. O. Macchiavelli, R. C. Pardo, A. Richard, M. A. Riley, G. Savard, M. Scheck, D. Seweryniak, M. K. Smith, R. Vondrasek, and A. Wiens, Phys. Rev. Lett. 116, 112503 (2016).

[18] B. Bucher, S. Zhu, C. Y. Wu, R. V. F. Janssens, R. N. Bernard, L. M. Robledo, T. R. Rodríguez, D. Cline, A. B. Hayes, A. D. Ayangeakaa, M. Q. Buckner, C. M. Campbell, M. P. Carpenter, J. A. Clark, H. L. Crawford, H. M. David, C. Dickerson, J. Harker, C. R. Hoffman, B. P. Kay, F. G. Kondev, T. Lauritsen, A. O. Macchiavelli, R. C. Pardo, G. Savard, D. Seweryniak, and R. Vondrasek, Phys. Rev. Lett. 118, 152504 (2017).

[19] G. A. Leander, W. Nazarewicz, P. Olanders, I. Ragnarsson, and J. Dudek, Phys. Lett. B 152, 284 (1985).

[20] A. N. Andreyev, J. Elseviers, M. Huyse, P. Van Duppen, S. Antalic, A. Barzakh, N. Bree, T. E. Cocolios, V. F. Comas, J. Diriken, D. Fedorov, V. Fedosseev, S. Franchoo, J. A. Heredia, O. Ivanov, U. Köster, B. A. Marsh, K. Nishio, R. D. Page, N. Patronis, M. Seliverstov, I. Tsekhanovich, P. Van den Bergh, J. Van De Walle, M. Venhart, S. Vermote, M. Veselsky, C. Wagemans, T. Ichikawa, A. Iwamoto, P. Möller, and A. J. Sierk, Phys. Rev. Lett. 105, 252502 (2010).

[21] L. Ghys, A. N. Andreyev, M. Huyse, P. Van Duppen, S. Sels, B. Andel, S. Antalic, A. Barzakh, L. Capponi, T. E. Cocolios, X. Derkx, H. De Witte, J. Elseviers, D. V. Fedorov, V. N. Fedosseev, F. P. Hessberger, Z. Kalaninová, U. Köster, J. F. W. Lane, V. Liberati, K. M. Lynch, B. A. Marsh, S. Mitsuoka, P. Möller, Y. Nagame, K. Nishio, S. Ota, D. Pauwels, R. D. Page, L. Popescu, D. Radulov, M. M. Rajabali, J. Randrup, E. Rapisarda, S. Rothe, K. Sandhu, M. D. Seliverstov, A. M. Sjödin, V. L. Truesdale, C. Van Beveren, P. Van den Bergh, Y. Wakabayashi, and M. Warda, Phys. Rev. C 90, 041301 (2014).

[22] K. Nishio, A. Andreyev, R. Chapman, X. Derkx, C. Düllmann, L. Ghys, F. Hessberger, K. Hirose, H. Ikezoe, J. Khuyagbaatar, B. Kindler, B. Lommel, H. Makii, I. Nishinaka, T. Ohtsuki, S. Pain, R. Sagaidak, I. Tsekhanovich, M. Venhart, Y. Wakabayashi, and S. Yan, Phys. Lett. B 748, 89 (2015).

[23] E. Prasad, D. J. Hinde, K. Ramachandran, E. Williams, M. Dasgupta, I. P. Carter, K. J. Cook, D. Y. Jeung, D. H. Luong, S. McNeil, C. S. Palshetkar, D. C. Rafferty, C. Simenel, A. Wakhle, J. Khuyagbaatar, C. E. Düllmann, B. Lommel, and B. Kindler, Phys. Rev. C 91, 064605 (2015).

[24] R. Tripathi, S. Sodaye, K. Sudarshan, B. K. Nayak, A. Jhingan, P. K. Pujari, K. Mahata, S. Santra, A. Saxena, E. T. Mirgule, and R. G. Thomas, Phys. Rev. C 92, 024610 (2015).

[25] J.-F. Martin, J. Taieb, A. Chatillon, G. Bélier, G. Boutoux, A. Ebran, T. Gorbinet, L. Grente, B. Laurent, E. Pellereau et al., Eur. Phys. J. A 51, 174 (2015).

[26] J. L. Rodríguez-Sánchez, J. Benlliure, J. Taïeb, H. Alvarez-Pol, L. Audouin, Y. Ayyad, G. Bélier, G. Boutoux, E. Casarejos, A. Chatillon, D. Cortina-Gil, T. Gorbinet, A. Heinz, A. Kelić-Heil, B. Laurent, J.-F. Martin, C. Paradela, E. Pellereau, B. Pietras, D.
Ramos, C. Rodríguez-Tajes, D. M. Rossi, H. Simon, J. Vargas, and B. Voss, Phys. Rev. C 94, 061601(R) (2016).

[27] T. Ichikawa, A. Iwamoto, P. Möller, and A. J. Sierk, Phys. Rev. C 86, 024610 (2012).

[28] P. Möller, J. Randrup, and A. J. Sierk, Phys. Rev. C 85, 024306 (2012).

[29] C. Schmitt, K. Pomorski, B. Nerlo-Pomorska, and J. Bartel, Phys. Rev. C 95, 034612 (2017).

[30] M. Warda, A. Staszczak, and W. Nazarewicz, Phys. Rev. C 86, 024601 (2012).

[31] S. Panebianco, J.-L. Sida, H. Goutte, J.-F. Lemaître, N. Dubray, and S. Hilaire, Phys. Rev. C 86, 064601 (2012).

[32] A. V. Andreev, G. G. Adamian, and N. V. Antonenko, Phys. Rev. C 86, 044315 (2012).

[33] A. V. Andreev, G. G. Adamian, N. V. Antonenko, and A. N. Andreyev, Phys. Rev. C 88, 047604 (2013).

[34] A. V. Andreev, G. G. Adamian, and N. V. Antonenko, Phys. Rev. C 93, 034620 (2016).

[35] J.-F. Lemaître, S. Goriely, S. Hilaire, and J.-L. Sida, Phys. Rev. C 99, 034612 (2019).

[36] T. Ichikawa and P. Möller, Phys. Lett. B 789, 679 (2019).

[37] H. Flocard, P. Quentin, D. Vautherin, M. Veneroni, and A. Kerman, Nucl. Phys. A 231, 176 (1974).

[38] M. Warda, J. L. Egido, L. M. Robledo, and K. Pomorski, Phys. Rev. C 66, 014310 (2002).

[39] L. Bonneau, Phys. Rev. C 74, 014301 (2006).

[40] N. Dubray, H. Goutte, and J.-P. Delaroche, Phys. Rev. C 77, 014310 (2008).

[41] J. C. Pei, W. Nazarewicz, J. A. Sheikh, and A. K. Kerman, Phys. Rev. Lett. 102, 192501 (2009).

[42] A. Staszczak, A. Baran, J. Dobaczewski, and W. Nazarewicz, Phys. Rev. C 80, 014309 (2009).

[43] J. D. McDonnell, W. Nazarewicz, and J. A. Sheikh, Phys. Rev. C 87, 054327 (2013).

[44] J. D. McDonnell, W. Nazarewicz, J. A. Sheikh, A. Staszczak, and M. Warda, Phys. Rev. C 90, 021302(R) (2014).

[45] R. Bernard, S. A. Giuliani, and L. M. Robledo, Phys. Rev. C 99, 064301 (2019).

[46] C. Simenel and A. S. Umar, Phys. Rev. C 89, 031601(R) (2014).

[47] G. Scamps, C. Simenel, and D. Lacroix, Phys. Rev. C 92, 011602(R) (2015).

[48] Y. Tanimura, D. Lacroix, and G. Scamps, Phys. Rev. C 92, 034601 (2015).

[49] P. M. Goddard, P. D. Stevenson, and A. Rios, Phys. Rev. C 92, 054610 (2015).

[50] P. M. Goddard, P. D. Stevenson, and A. Rios, Phys. Rev. C 93, 014620 (2016).

[51] A. Bulgac, P. Magierski, K. J. Roche, and I. Stetcu, Phys. Rev. Lett. 116, 122504 (2016).

[52] C. Simenel and A. S. Umar, Prog. Part. Nucl. Phys. 103, 19 (2018).

[53] A. Bulgac, S. Jin, K. J. Roche, N. Schunck, and I. Stetcu, Phys. Rev. C 100, 034615 (2019).

[54] N. Schunck and L. M. Robledo, Rep. Prog. Phys. 79, 116301 (2016).

[55] See Supplemental Material at http://link.aps.org/supplemental/ 10.1103/PhysRevC.100.041602 for details of the method and additional calculations.

[56] Ka-Hae Kim, Takaharu Otsuka, and Paul Bonche, J. Phys. G 23, 1267 (1997).

[57] G. Scamps and D. Lacroix, Phys. Rev. C 87, 014605 (2013). 
[58] P. Bonche, H. Flocard, and P. H. Heenen, Comput. Phys. Commun. 171, 49 (2005).

[59] H. Tao, J. Zhao, Z. P. Li, T. Nikšić, and D. Vretenar, Phys. Rev. C 96, 024319 (2017).

[60] J. Zhao, T. Niksic, D. Vretenar, and S.-G. Zhou, Phys. Rev. C 99, 014618 (2019).

[61] J. Zhao, J. Xiang, Z.-P. Li, T. Nikšić, D. Vretenar, and S.-G. Zhou, Phys. Rev. C 99, 054613 (2019).

[62] H. Goutte, J. F. Berger, P. Casoli, and D. Gogny, Phys. Rev. C 71, 024316 (2005).

[63] D. Regnier, N. Dubray, M. Verrière, and N. Schunck, Comput. Phys. Commun. 225, 180 (2018).

[64] D. Regnier, N. Dubray, and N. Schunck, Phys. Rev. C 99, 024611 (2019).

[65] P. Möller and J. Randrup, Phys. Rev. C 91, 044316 (2015).

[66] J. Sadhukhan, W. Nazarewicz, and N. Schunck, Phys. Rev. C 93, 011304(R) (2016).

[67] Y. Tanimura, D. Lacroix, and S. Ayik, Phys. Rev. Lett. 118, 152501 (2017).

[68] A. D. Becke and K. E. Edgecombe, J. Chem. Phys. 92, 5397 (1990).

[69] P.-G. Reinhard, J. A. Maruhn, A. S. Umar, and V. E. Oberacker, Phys. Rev. C 83, 034312 (2011).

[70] P. A. Butler and W. Nazarewicz, Rev. Mod. Phys. 68, 349 (1996).

[71] L. M. Robledo and G. F. Bertsch, Phys. Rev. C 84, 054302 (2011).

[72] P. A. Butler, J. Phys. G 43, 073002 (2016).

[73] W. Nazarewicz, J. Dudek, R. Bengtsson, T. Bengtsson, and I. Ragnarsson, Nucl. Phys. A 435, 397 (1985).

[74] M. G. Itkis, V. N. Okolovich, and G. N. Smirenkin, Nucl. Phys. A 502, 243 (1989).
[75] I. Tsekhanovich, A. Andreyev, K. Nishio, D. Denis-Petit, K. Hirose, H. Makii, Z. Matheson, K. Morimoto, K. Morita, W. Nazarewicz, R. Orlandi, J. Sadhukhan, T. Tanaka, M. Vermeulen, and M. Warda, Phys. Lett. B 790, 583 (2019).

[76] A. O. Macchiavelli, J. Burde, R. M. Diamond, C. W. Beausang, M. A. Deleplanque, R. J. McDonald, F. S. Stephens, and J. E. Draper, Phys. Rev. C 38, 1088 (1988).

[77] D. N. Poenaru, R. A. Gherghescu, and W. Greiner, Phys. Rev. Lett. 107, 062503 (2011).

[78] Y. L. Zhang and Y. Z. Wang, Phys. Rev. C 97, 014318 (2018).

[79] K. P. Santhosh and C. Nithya, Phys. Rev. C 97, 064616 (2018).

[80] M. Warda, A. Zdeb, and L. M. Robledo, Phys. Rev. C 98, 041602(R) (2018).

[81] Z. Matheson, S. A. Giuliani, W. Nazarewicz, J. Sadhukhan, and N. Schunck, Phys. Rev. C 99, 041304(R) (2019).

[82] A. Wakhle, C. Simenel, D. J. Hinde, M. Dasgupta, M. Evers, D. H. Luong, R. du Rietz, and E. Williams, Phys. Rev. Lett. 113, 182502 (2014).

[83] A. S. Umar, V. E. Oberacker, and C. Simenel, Phys. Rev. C 94, 024605 (2016).

[84] K. Sekizawa and K. Yabana, Phys. Rev. C 93, 054616 (2016).

[85] L. Guo, C. Shen, C. Yu, and Z. Wu, Phys. Rev. C 98, 064609 (2018).

[86] K. Sekizawa, Front. Phys. 7, 20 (2019).

[87] M. Morjean, D. J. Hinde, C. Simenel, D. Y. Jeung, M. Airiau, K. J. Cook, M. Dasgupta, A. Drouart, D. Jacquet, S. Kalkal, C. S. Palshetkar, E. Prasad, D. Rafferty, E. C. Simpson, L. Tassan-Got, K. Vo-Phuoc, and E. Williams, Phys. Rev. Lett. 119, 222502 (2017). 